\title{
Detection of the extra tRNA genes in mitochondrial genomes of amphipods using lineage-specific models
}

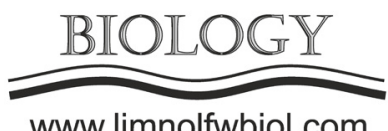

\author{
Romanova E.V. ${ }^{1 *}$, Bukin Y.S. ${ }^{1,2}$, Mikhailov K.V. ${ }^{3,4}$, Logacheva M.D. ${ }^{3,4}$, \\ Aleoshin V.V. ${ }^{3,4}$, Sherbakov D.Yu. ${ }^{1,2}$ \\ ${ }^{1}$ Limnological Institute, Siberian Branch of the Russian Academy of Sciences, Ulan-Batorskaya Str., 3, Irkutsk, 664033, Russia \\ ${ }^{2}$ Faculty of Biology and Soil Studies, Irkutsk State University, Sukhe-Bator Str., 5, Irkutsk, 664011, Russia \\ ${ }^{3}$ Belozersky Institute for Physicochemical Biology, Lomonosov Moscow State University, Moscow, Leninskye gory, house 1, building 40, \\ 119992, Russia \\ ${ }^{4}$ Institute for Information Transmission Problems, Bolshoy Karetny per. 19, building 1, Moscow, 127051, Russia
}

\begin{abstract}
We developed and applied models for tRNA genes prediction in mitochondrial (mt) genomes of amphipods. Using these models, we refined annotation of available mt genomes of amphipod species by finding 16 new tRNA genes in total. Phylogenetic inference and genetic distance analysis allowed to verify these findings and to identify cases of tRNA gene remolding. The majority remolded tRNA genes (seven out of ten) were found in mt genomes of Baikalian amphipods. The effectivity of amphipod-specific models for tRNA genes prediction in MiTFi program implies potential usefulness of other lineage-specific tRNA models for correct annotation of mt genomes of other organisms.
\end{abstract}

Keywords: tRNA gene, mitochondrial genome, tRNA gene remolding, amphipods, Baikal

\section{Introduction}

In the last decade mitochondrial (mt) genome became a widely used tool for phylogenetic and evolutionary studies. However, the annotation of mt genomes submitted in databases is not always trustworthy due to imperfect performance of annotation programs. For instance, the prediction of tRNA genes in different lineages is complicated by insufficient specificity of tRNA models implemented in annotation programs. In addition, all of them do not identify tRNA genes underwent to remolding (changing tRNA specify throw mutation in their anticodon) (Cantatore et al., 1987). Our recent work showed some cases of duplicated and presumably remolded tRNA genes in the $\mathrm{mt}$ genomes of Baikalian amphipods (Romanova et al., 2016). In the current study, we performed refinement of tRNA gene annotation in all available mt genomes of amphipods using different bioinformatics approaches.

\section{Material and methods}

Using Infernal software, we developed models performed more specific identification of tRNA genes in $\mathrm{mt}$ genomes of amphipods than default metazoan models implemented in MiTFi annotation tool (Jühling et al., 2012). The identity of newly found tRNA genes was verified using phylogenetic inference and genetic pairwise analysis performed in $\mathrm{R}$.

\section{Results}

We found 17 cases of tRNA genes duplications in $\mathrm{mt}$ genomes of amphipods where ten of them were tRNA genes underwent to remolding. Notably, nine cases of tRNA genes duplications were found in $\mathrm{mt}$ genomes of Baikalian amphipods, and in seven of them, tRNA gene remolding was detected.

\section{Discussion}

Consideration the topology of phylogenetic tree and gene orders in studied $\mathrm{mt}$ genomes allowed to predict scenarios of tRNA genes rearrangement in amphipod species. tRNA gene duplication and remolding events arose independently in different species. In the majority of Baikalian amphipods, tRNA genes order differ from their deduced ancestral gene order pattern identical to gen. Gammarus, that indicates the active rearrangement process typical for $\mathrm{mt}$ genes in Baikalian amphipods.

\section{Conclusions}

1. Amphipod-specific models showed better performance for prediction of tRNA genes in amphipods than commonly used metazoan models implemented in MiTFi. 
2. In mt genomes of amphipod we identified and verified 16 additional tRNA genes, ten of them underwent to remolding.

3. The most duplicated and remolded tRNA genes were found in mt genomes of six Baikalian amphipod species.

\section{Acknowledgements}

This study was supported by the governmentally funded project 0345-2019-0004 (AAAA-A16$116122110060-9)$ and by RFBR grant No. 19-05-00398.

\section{References}

Cantatore P., Gadaleta M.N., Roberti M. et al. 1987. Duplication and remoulding of tRNA genes during the evolutionary rearrangement of mitochondrial genomes. Nature 329: 853-855. DOI: 10.1038/329853a0

Jühling F., Pütz J., Bernt M. et al. 2012. Improved systematic tRNA gene annotation allows new insights into the evolution of mitochondrial tRNA structures and into the mechanisms of mitochondrial genome rearrangements. Nucleic Acids Research 40: 2833-2845. DOI: 10.1093/nar/ gkr1131

Romanova E.V., Aleoshin V.V., Kamaltynov R.M. et al. 2016. Evolution of mitochondrial genomes in Baikalian amphipods. BMC Genomics 17. DOI: 10.1186/ s12864-016-3357-z 